\title{
Signed Networks in Social Media
}

\author{
Jure Leskovec \\ Stanford University \\ jure@cs.stanford.edu
}

\author{
Daniel Huttenlocher \\ Cornell University \\ dph@cs.cornell.edu
}

\author{
Jon Kleinberg \\ Cornell University \\ kleinber@cs.cornell.edu
}

\begin{abstract}
Relations between users on social media sites often reflect a mixture of positive (friendly) and negative (antagonistic) interactions. In contrast to the bulk of research on social networks that has focused almost exclusively on positive interpretations of links between people, we study how the interplay between positive and negative relationships affects the structure of on-line social networks. We connect our analyses to theories of signed networks from social psychology. We find that the classical theory of structural balance tends to capture certain common patterns of interaction, but that it is also at odds with some of the fundamental phenomena we observe - particularly related to the evolving, directed nature of these on-line networks. We then develop an alternate theory of status that better explains the observed edge signs and provides insights into the underlying social mechanisms. Our work provides one of the first large-scale evaluations of theories of signed networks using on-line datasets, as well as providing a perspective for reasoning about social media sites.
\end{abstract}

\section{Author Keywords}

signed networks, structural balance, status theory, positive edges, negative edges, trust, distrust.

\section{ACM Classification Keywords}

H.5.3 Information Systems: Group and Organization Interfaces-Web-based interaction.

\section{General Terms \\ Human Factors, Measurement, Design.}

\section{INTRODUCTION}

Social network analysis provides a useful perspective on a range of social computing applications. The structure of networks arising in such applications offers insights into patterns of interactions, and reveals global phenomena at scales that may be hard to identify when looking at a finer-grained resolution. At the same time, there is an ongoing challenge in adapting such network approaches to the study of social computing: users develop rich relationships with one another in these settings, while network analyses generally re-

Permission to make digital or hard copies of all or part of this work for personal or classroom use is granted without fee provided that copies are not made or distributed for profit or commercial advantage and that copies bear this notice and the full citation on the first page. To copy otherwise, or republish, to post on servers or to redistribute to lists, requires prior specific permission and/or a fee.

CHI 2010, April 10 - 15, 2010, Atlanta, Georgia, USA

Copyright 2010 ACM 978-1-60558-929-9/10/04...\$10.00. duce these complex relationship to the existence of simple pairwise links. It is a fundamental research problem to bridge the gap between the richness of the existing relationships and the stylized nature of network representations of these relationships.

The main focus of our work here is to examine the interplay between positive and negative links in social media a dimension of on-line social network analysis that has been largely unexplored. With relatively few exceptions (e.g., [1, $15,16])$, research in on-line social networks has focused on contexts in which the interactions have largely only positive interpretations - that is, connecting people to their friends, fans, followers, and collaborators. But in many settings it is important to also explicitly take negative relations into consideration, especially when studying interactions in social media: discussion lists are filled with controversy and disagreement, and social-networking sites harbor antagonism alongside amity. The richness of a social network in such cases generally consists of a mixture of both positive and negative interactions, co-existing in a single structure.

We aim to develop a better understanding of the role that network structure plays when some links between people are positive while others are negative. For instance, in on-line rating sites such as Epinions, people can give both positive and negative ratings not only to items but also to other raters. In on-line discussion sites such as Slashdot, users can tag other users as "friends" and "foes". Our approach here is to adapt and extend theories from social psychology to analyze these types of signed networks as they arise in social computing applications. These theories enable us to characterize the differences between the observed and predicted configurations of positive and negative links in on-line social networks. We also use contrasts between the theories to draw inferences about how links are being used in particular social computing applications. In addition to insights into the applications themselves, our studies provide, to the best of our knowledge, some of the first large-scale evaluations of these social-psychological theories via on-line datasets.

Positive and negative links in on-line data. To carry out such an investigation, we need two fundamental ingredients: (i) large-scale datasets from social applications where the sign of each link - whether it is positive or negative - can be reliably determined, and (ii) theories of signed networks that help us reason about how different patterns of positive and negative links provide evidence for the expression of different kinds of relationships across these applications. 


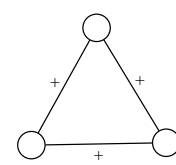

$\operatorname{triad} T_{3}$

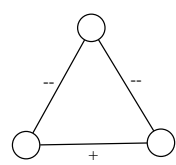

$\operatorname{triad} T_{1}$

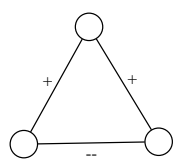

$\operatorname{triad} T_{2}$

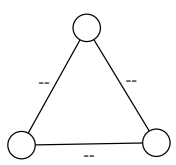

$\operatorname{triad} T_{0}$
Figure 1. Undirected signed triads. Based on the number of positive edges we label triads with odd number of pluses as balanced $\left(T_{3}, T_{1}\right)$, and triads with even positive edges $\left(T_{2}, T_{0}\right)$ as unbalanced.

We investigate social network structures from three widelyused Web sites. The first is the trust network of Epinions, where users create signed directed relations to each other indicating trust or distrust. The second is the social network of the technology blog Slashdot, where users designate others as "friends" or "foes." The third is the network defined by votes for Wikipedia admin candidates. When a Wikipedia user is considered for a promotion to the status of an admin, the community is able to cast public votes in favor of or against the promotion of this admin candidate. We view a positive vote as corresponding to a positive link from the voter to the candidate, and a negative vote as a negative link. The Epinions and Slashdot networks are explicitly presented to users as social networking features of the sites, whereas in the case of Wikipedia the network interpretation is implicit.

The meanings of positive and negative signs are different across these settings, and this is precisely the point: we wish to use theories of signed edges to evaluate how the positive and negative edges are being used in each setting, and to identify commonalities and differences in the underlying networks in relatively different application contexts. Moreover, while the current work focuses on domains in which the signs of edges are overtly denoted (either explicitly by direct linking, or implicitly through actions such as voting on Wikipedia), we believe the underlying issues reach more broadly into any application where positive and negative attitudes between users can be conveyed, such as through sentiment in text [20].

Theories of signed networks: Balance. We analyze these on-line signed networks using two different theories, and a central issue in our study is the extent to which each of these theories provides a plausible explanation for the structure and dynamics of the observed networks.

The first of these theories is structural balance theory, which originated in social psychology in the mid-20th-century. As formulated by Heider in the 1940s [14], and subsequently cast in graph-theoretic language by Cartwright and Harary [4], structural balance considers the possible ways in which triangles on three individuals can be signed, and posits that triangles with three positive signs (three mutual friends, Figure $1 T_{3}$ ) and those with one positive sign (two friends with a common enemy, Fig. $1 T_{1}$ ) are more plausible — and hence should be more prevalent in real networks - than triangles with two positive signs (two enemies with a common friend, $T_{2}$ ) or none (three mutual enemies, $T_{0}$ ). Balanced triangles with three positive edges exemplify the principle that "the friend of my friend is my friend," whereas those with one positive and two negative edges capture the notions that "the friend of my enemy is my enemy," "the enemy of my friend is my enemy," and "the enemy of my enemy is my friend."
Structural balance theory has been developed extensively in the time since this initial work [21], including the formulation of a variant - weak structural balance - proposed by Davis in the 1960s as a way of eliminating the assumption that "the enemy of my enemy is my friend" [7]. In particular, weak structural balance posits that only triangles with exactly two positive edges are implausible in real networks, and that all other kinds of triangles should be permissible.

Theories of signed networks: Status. Balance theory can be viewed as a model of likes and dislikes. However, as Guha et al. observe in the context of Epinions [13], a signed link from $A$ to $B$ can have more than one possible interpretation, depending on $A$ 's intention in creating the link. In particular, a positive link from $A$ may mean, " $B$ is my friend," but it also may mean, "I think $B$ has higher status than I do." Similarly, a negative link from $A$ to $B$ may mean " $B$ is my enemy" or "I think $B$ has lower status than I do."

Here we develop this idea into a new theory of status, which provides a different organizing principle for directed networks of signed links. In this theory of status, we consider a positive directed link to indicate that the creator of the link views the recipient as having higher status; and a negative directed link indicates that the recipient is viewed as having lower status. These relative levels of status can then be propagated along multi-step paths of signed links, often leading to different predictions than balance theory.

Comparing the two theories. To give a sense for how the differences between status and balance arise, consider the situation in which a user $A$ links positively to a user $B$, and $B$ in turn links positively to a user $C$. If $C$ then forms a link to $A$, what sign should we expect this link to have? Balance theory predicts that since $C$ is a friend of $A$ 's friend, we should see a positive link from $C$ to $A$. Status theory, on the other hand, predicts that $A$ regards $B$ as having higher status, and $B$ regards $C$ as having higher status - so $C$ should regard $A$ as having low status and hence be inclined to link negatively to $A$. In other words, the two theories suggest opposite conclusions in this case.

Thus balance theory predicts that certain types of triads such as all-positive cycles should be overrepresented compared to chance, whereas status theory makes predictions that often differ. We study all the possible types of signed triads and the predictions made by the different theories. In doing so we consider several experimental conditions, including both directed and undirected networks, as well as both respecting and ignoring the order in which edges were created. For each such experimental condition we consider whether the observed number of triads of each type is overrepresented or underrepresented compared to chance, and contrast that with the predictions made by the balance and status theories. This analysis give us a picture of the aggregate patterns of links in the social networks, and the degree to which they are explained in terms of each theory.

Summary of Findings: Comparison of Balance and Status. Both of these theories concern relationships between people; by adapting them to our on-line network datasets, 
they provide potentially informative perspectives on the link structures we find there.

Balance theory was initially intended as a model for undirected networks, although it has been commonly applied to directed networks by simply disregarding the directions of the links [21]. When we do this, we find significant alignment between the observed network data and Davis's notion of weak structural balance: triangles with exactly two positive edges are massively underrepresented in the data relative to chance, while triangles with three positive edges are massively overrepresented. In two of the three datasets, triangles with three negative edges are also overrepresented, which is at odds with Heider's formulation of balance theory. These findings are already intriguing, since it has traditionally been difficult to evaluate the predictions of structural balance theory on large network datasets. Rather, empirical investigations to date have generally focused on small networks where social relations can be observed through direct interaction with the individuals involved (see e.g. [8]). The trouble with assessing structural balance at small scales is that one expects its predictions to be aggregate rather than absolute - that is, one expects to see certain kinds of triangles as statistically more abundant or less abundant in the data, and the significance of such biases towards certain kinds of triangles can stand out much more clearly when they are accumulated over a large amount of data.

Ultimately, however, we would like to understand the networks in these on-line systems as directed structures that evolve over time. When we view the network data in this way, our main conclusion is that the theory of status is more effective at explaining local patterns of signed links, and that it naturally extends to capture richer aspects of user behavior, including heterogeneity in their linking tendencies. For example in the case offered as an illustration above, where user $A$ links positively to user $B$ and user $B$ links positively to user $C$, we find that negative links from $C$ to $A$ are massively overrepresented relative to chance, with positive links correspondingly underrepresented.

Implications. There are several potentially interesting implications of our results. First, the comparison of balance and status provides insights into ways in which people use linking mechanisms in social computing applications. In particular, there are important domains such as rating reviewers on Epinions and voting for admins on Wikipedia in which such links appear, in aggregate, to be used more dominantly for expressions of status than for expressions of likes and dislikes.

The contrast between balance and status is also related to the distinction between undirected and directed interpretations of links. Our findings suggest that it is important to understand the roles of different theories in both undirected and directed representations of networks. Indeed, the theory of status only makes sense with directed links - since it posits a status differential from the creator of a link to its recipient - while the theory of balance has been applied in both undirected and directed settings (e.g., [21]). The fact that (weak) balance is broadly consistent with the undirected representa- tion of our network data, while status is more consistent with the directed representation, shows that it possible for different theories to be appropriate to different levels of resolution in the representation of a single network.

In the final part of the paper, we describe further structural investigations that provide insight into ways in which signed links are used in these applications. First, we find that aspects of the theory of balance hold more strongly on the subset of links in these networks that are reciprocated consisting of directed links in both directions between two users. This suggests that reciprocal link formation may follow a different pattern of use in these systems than unreciprocated link formation. However, it is important to note that such reciprocal relations account for only a small proportion of the links between people on these sites.

Second, we find a connection between the sign of a link and the extent to which it is embedded [12], i.e., with the two endpoints having links to many common neighbors. A link is significantly more likely to be positive when its two endpoints have multiple neighbors (of either sign) in common. This observation is consistent with qualitative notions of social capital $[3,5]$ - users with common neighbors have relations that are "on display" in a social sense, and hence have greater implicit pressure to remain positive. Indeed in the three different social applications that we study, this effect is strongest in the case of voting for Wikipedia admins, which is the setting that makes the relations most prominently visible to users. This suggests some of the ways in which the presence of common neighbors, and more overt forms of public display, can have an effect on the use of signed links.

These findings about aggregate structural properties also begin to address a broad and largely open issue, which is to understand the sources of individual variation in linking behavior. While reciprocation and embeddedness are only two dimensions along which to explore such variation, we believe that the definitions and analysis pursued here can help in framing further investigation of questions regarding individual variation.

\section{RELATED WORK}

There is by now a large and rapidly growing literature on the analysis of social networks arising in on-line domains [18]; as we noted at the outset, this line of work has almost exclusively treated networks as implicitly having positive signs only. For example, portions of our analysis can be viewed as variants on the problem of link prediction [17] and tiestrength prediction [10], but in each case adapted to take the signs of links into account.

Two recent papers in the analysis of on-line social networks stand out as taking the signs of links into account. Brzozowski et al. study the positive and negative relationships that exist on ideologically oriented sites such as Essembly [1], but with the goal of predicting outcomes of group votes rather than the broader organization of the social network. Kunegis et al. study the friend/foe relationships on Slashdot, and compute global network properties [15], but do not evaluate theories of balance and status as we do here. 


\begin{tabular}{l||r|r|r} 
& Epinions & Slashdot & Wikipedia \\
\hline Nodes & 119,217 & 82,144 & 7,118 \\
Edges & 841,200 & 549,202 & 103,747 \\
+ edges & $85.0 \%$ & $77.4 \%$ & $78.7 \%$ \\
- edges & $15.0 \%$ & $22.6 \%$ & $21.2 \%$ \\
Triads & $13,375,407$ & $1,508,105$ & 790,532 \\
\multicolumn{3}{c}{ Table 1. Dataset statistics. }
\end{tabular}

\begin{tabular}{l|l} 
Symbol & Meaning \\
\hline$T_{i}$ & Signed triad, also the number of triads of type $T_{i}$ \\
$\Delta$ & Total number of triads in the network \\
$p$ & Fraction of positive edges in the network \\
$p\left(T_{i}\right)$ & Fraction of triads $T_{i}, p\left(T_{i}\right)=T_{i} / \Delta$ \\
$p_{0}\left(T_{i}\right)$ & A priori prob. of $T_{i}($ based on sign distribution) \\
$E\left[T_{i}\right]$ & Expected number of triads $T_{i}, E\left[T_{i}\right]=p_{0}\left(T_{i}\right) \Delta$ \\
$s\left(T_{i}\right)$ & Surprise, $s\left(T_{i}\right)=\left(T_{i}-E\left[T_{i}\right]\right) / \sqrt{\Delta p_{0}\left(T_{i}\right)\left(1-p_{0}\left(T_{i}\right)\right)}$
\end{tabular}

Table 2. Table of symbols.

There are also large bodies of work involving negative relationships in on-line domains that pursue directions different from our network focus here. One line of work focuses on norms to control deviant behavior in on-line communities (e.g. [6] and the references therein). In a different direction, a large body of recent work in sentiment analysis [20] has studied on-line textual data in which individuals can express both positive and negative attitudes toward one another, but without addressing the consequences for network structure.

The datasets we study here have also been investigated by researchers for other purposes. Guha et al. study the trust network of Epinions [13]. Lampe et al. study the user rating mechanisms on Slashdot [16]. Burke and Kraut study the voting process that produces our Wikipedia signed network [2], but with the goal of modeling election outcomes.

Finally, the notion of status plays a role in many lines of work in the social sciences, such as the role that behaviorstatus theory plays in social exchange theory [9, 22]. However, these notions are distinct from the ways in which we formulate definitions of status as a counterpart to balance in signed directed networks.

\section{DATASET DESCRIPTION}

As described above, we consider three large online social networks where links are explicitly positive or negative: (i) the trust network of the Epinions product review Web site, where users can indicate their trust or distrust of the reviews of others; (ii) the social network of the blog Slashdot, where a signed link indicates that one user likes or dislikes the comments of another; and (iii) the voting network of Wikipedia, where a signed link indicates a positive or negative vote by one user on the promotion to admin status of another.

Table 1 gives statistics for all three datasets. Our networks have on the approximate order of tens to hundreds of thousand nodes, and less than a million edges. In each network the edges are inherently directed, since we know which user created the edge. In all networks the background proportion of positive edges is about the same, with roughly $80 \%$ of the edges having a positive sign.

\section{ANALYSIS OF UNDIRECTED NETWORKS}

We begin by analyzing the network data in an undirected representation, where we do not take the directions of links

\begin{tabular}{|c|c|c|c|c|c|}
\hline \multicolumn{2}{|c|}{$\operatorname{Triad} T_{i}$} & $\left|T_{i}\right|$ & $p\left(T_{i}\right)$ & $p_{0}\left(T_{i}\right)$ & $s\left(T_{i}\right)$ \\
\hline \multicolumn{6}{|c|}{ Epinions } \\
\hline$T_{3}$ & +++ & $11,640,257$ & 0.870 & 0.621 & 1881.1 \\
\hline$T_{1}$ & +-- & 947,855 & 0.071 & 0.055 & 249.4 \\
\hline$T_{2}$ & ++- & 698,023 & 0.052 & 0.321 & -2104.8 \\
\hline$T_{0}$ & --- & 89,272 & 0.007 & 0.003 & 227.5 \\
\hline \multicolumn{6}{|c|}{ Slashdot } \\
\hline$T_{3}$ & +++ & $1,266,646$ & 0.840 & 0.464 & 926.5 \\
\hline$T_{1}$ & +-- & 109,303 & 0.072 & 0.119 & -175.2 \\
\hline$T_{2}$ & ++- & 115,884 & 0.077 & 0.406 & -823.5 \\
\hline$T_{0}$ & --- & 16,272 & 0.011 & 0.012 & -8.7 \\
\hline \multicolumn{6}{|c|}{ Wikipedia } \\
\hline$T_{3}$ & +++ & 555,300 & 0.702 & 0.489 & 379.6 \\
\hline$T_{1}$ & +-- & 163,328 & 0.207 & 0.106 & 289.1 \\
\hline$T_{2}$ & ++- & 63,425 & 0.080 & 0.395 & -572.6 \\
\hline$T_{0}$ & --- & 8,479 & 0.011 & 0.010 & 10.8 \\
\hline
\end{tabular}

Table 3. Number of balanced and unbalanced undirected triads.

into account. In this context, we can evaluate the predictions of structural balance theory by considering the frequencies of different types of signed triads - sets of three nodes with signed edges among all pairs.

Table 3 gives the counts of the four possible signed undirected triads, while Table 2 summarizes the symbols we use throughout the paper. Let $p$ denote the fraction of positive edges in the network. The four possible signed undirected triads are denoted $T_{0}, T_{1}, T_{2}$, and $T_{3}$ (Figure 1). Among all triads in the data, the number that are of type $T_{i}$ is denoted $\left|T_{i}\right|$ and the fraction of type $T_{i}$ is denoted $p\left(T_{i}\right)$. Now, we would like to compare how this empirical frequency of triad types compares to the corresponding frequencies if edge signs were produced at random from the same background distribution of positive and negative signs. Thus, we shuffle the signs of all edges in the graph (keeping the fraction $p$ of positive edges the same), and we let $p_{0}\left(T_{i}\right)$ denote the expected fraction of triads that are of type $T_{i}$ after this shuffling.

If $p\left(T_{i}\right)>p_{0}\left(T_{i}\right)$, then triads of type $T_{i}$ are overrepresented in the data relative to chance; if $p\left(T_{i}\right)<p_{0}\left(T_{i}\right)$, then they are underrepresented. We also want to measure how significant this over- or underrepresentation is. Thus, we define the surprise $s\left(T_{i}\right)$ to be the number of standard deviations by which the actual quantity of type- $T_{i}$ triads differs from the expected number under the random-shuffling model.

Due to the Central Limit Theorem the distribution of $s\left(T_{i}\right)$ is approximately a standard normal distribution and so we would expect surprise on the order of tens to already be significant $\left(s\left(T_{i}\right)=6\right.$ gives a p-value of $\left.\approx 10^{-8}\right)$. However, the values of surprise we find in our data are typically much larger. This means that due to the scale of the data and the large number of triads almost all our observations are statistically significant with p-values practically equal to zero.

We find that the all-positive triad $T_{3}$ is heavily overrepresented in all three datasets, and the triad $T_{2}$ consisting of two enemies with a common friend is heavily underrepresented. Based on the relative magnitudes of $p\left(T_{i}\right)$ and $p_{0}\left(T_{i}\right)$, we see that $T_{3}$ tends to be over represented by about $40 \%$ in all three datasets. Similarly, the unbalanced triad $T_{2}$ is underrepresented by about $75 \%$ in Epinions and Slashdot and $50 \%$ in Wikipedia. These observations so far fit well into Heider's original notion of structural balance. 
However, the relative abundances of triad types $T_{1}$ (single positive edge) and $T_{0}$ (all negative edges) differ between the datasets, and none of the datasets follow Heider's theory in both having $T_{1}$ overrepresented and $T_{0}$ underrepresented. Thus, the picture is more consistent with Davis's weaker notion of balance, where $T_{2}$ is viewed as implausible but there is no a priori reason to favor one of $T_{1}$ or $T_{0}$ over the other.

\section{ANALYSIS OF EVOLVING DIRECTED NETWORKS}

We now consider the networks in these systems as directed graphs, incorporating the fact that the links being created go from one user to another, with the sign of a link from $A$ to $B$ being generated by $A$. In the introduction, we discussed how the theories of balance and status offer competing interpretations for how we should expect such directed links to be signed. For example, as noted there, positive cycles that is, directed triads with positive links from $A$ to $B$ to $C$ to $A-$ are underrepresented in the data. This conflicts with balance theory, but is consistent with status theory.

Timing and Diversity: Generative and Receptive Baselines. Beyond just the directionality of links, there are additional features of the data that we take into account when evaluating these models. First, links are created at specific points in time, so rather than thinking of directed triads as existing in a static snapshot of the network, we consider the order in which links are added to the network. Thus, we study how directed triads form, as follows. When a user $A$ links to a user $B$, suppose there is already a user $X$ with the property that $X$ has links to or from $A$, and also to or from $B$. This means there is a two-step semi-path from $A$ to $B$ through $X$ (a path in which the directions of the edges do not matter), and the formation of the $A-B$ link adds a shortcut to this path, producing a directed triad on $A, B$, and $X$.

Second, different users make use of positive and negative signs differently. At the most basic level, some users produce links almost exclusively of one sign or the other, while others produce a relatively even mix of both positive and negative links. We will refer to the overall fraction of positive signs that a user creates, considering all her links, as her generative baseline. Similarly, some users receive links that are almost exclusively of one sign or the other, while others receive a mix of signs. We will refer to the overall fraction of positive signs in the links a user receives as his receptive baseline. Given this, we should compare the abundance of positive and negative links to the generative and receptive baselines of the users producing and receiving these links.

Once we incorporate these aspects of the data, we discover further mysteries - beyond just the scarcity of positive cycles - that seem to call for alternatives to balance theory. For example, consider the case of joint positive endorsement - a situation in which a node $X$ links positively to each of two nodes $A$ and $B$. Suppose that in this case, $A$ now forms a link to $B$ (i.e., triad $t_{9}$ of Figure 2); should we expect there to be an elevated probability of the link being positive, or a reduced probability of the link being positive?

In fact, in our data, the question turns out to have a more subtle answer than either of these alternatives. The link that is produced in this situation is more likely to be positive than the generative baseline of $A$, but at the same time less likely to be positive than the receptive baseline of $B$. Balance theory, of course, makes a much more naive prediction: since $A$ and $B$ are both friends of $X$, they should be friends of each other. Can status theory explain this dual and opposite pair of deviations from the baselines of $A$ and $B$ ?

We now show that in fact it can, and explaining how this works forms the motivation for a theory of how status effects can influence the signs of directed links.

\section{Formulating a Theory of Status}

Since the phenomenon we are trying to capture is subtle but in the end familiar from everyday life, we begin with a hypothetical example to motivate the subsequent definitions.

A Motivating Example. Suppose we were to interview the players on a college soccer team: for certain players $A$, and certain teammates $B$ of $A$, we ask, "How do you think the skill of player $B$ compares to yours?" Suppose further that the players roughly agree on a ranking of each other by skill, which serves as an approximate (though not perfect) ranking of the team members by status. From the results of these interviews, we could produce a signed directed graph whose nodes are the players, and with a directed edge from $A$ to $B$ if we asked $A$ for her opinion of $B$. A positive link from $A$ to $B$ would indicate that $A$ thinks highly of $B$ 's skill relative to her own, while a negative link would indicate that $A$ thinks she is better than $B$.

If we were just given this signed directed graph, and knew nothing else about the soccer team, then we could still make inferences about the signs of links that we haven't yet observed, using the context provided by the rest of the network. Suppose for example that we are about to ask player $A$ 's opinion of another player $B$, but we don't currently have $A$ 's answer and hence don't yet know the sign of the link from $A$ to $B$. We can nonetheless make predictions about it from the links whose signs we do know, as follows. Suppose that we know from the data already collected that $A$ and $B$ have each received a positive evaluation from a third player $X$. Here is a pair of facts we could conjecture about the link from $A$ to $B$, given the positive links from $X$ to $A$ and $B$.

- Since $B$ has been positively evaluated by another team member, $B$ is more likely than not to have above-average skill. Therefore, the evaluation that $A$ gives $B$ should be more likely to be positive than an evaluation given by $A$ to a random team member.

- Since $A$ has been positively evaluated by another team member, $A$ is also more likely than not to have aboveaverage skill. Therefore, the evaluation that $A$ gives $B$ should be less likely to be positive than an evaluation received by $B$ from a random team member.

There are several subtleties here. First, we're using the indirection provided by a third party $X$ to make inferences about the relation between $A$ and $B$, based on assumptions about status. Second, the context provided by $X$ causes the sign of the $A-B$ link to deviate from a random baseline in different 
directions depending on whether we're looking at it from $A$ 's point of view or $B$ 's point of view. More precisely, since $B$ has above-average skill, $A$ will likely give $B$ a higher evaluation than $A$ would give to a random team member. On the other hand, since $A$ has above-average skill, $B$ is less likely to receive a positive evaluation from $A$ than she would receive from a random team member. Despite the complexity of these conclusions, they reflect genuine and natural properties of status ordering among a group of people. They also agree with our observations about joint positive endorsement in the data mentioned above.

We turn now to the data, where we will find that the users of these on-line networks create signed links in ways that correspond closely to the behavior of the players on our hypothetical soccer team. But extracting this finding from the data will require formulating a sequence of definitions that captures the intuition suggested by this example.

Contextualized Links. The first portion of our definitions capture the idea that we will evaluate the sign of a link created from $A$ to $B$ in the context of $A$ and $B$ 's relations to additional nodes $X$ with whom they have links. (For example, the node $X$ in our example who jointly endorses $A$ and $B$.) Thus, we define a contextualized link (more briefly, a c-link) to be a triple $(A, B ; X)$ with the the property that a link forms from $A$ to $B$ after each of $A$ and $B$ already has a link either to or from $X$. Overall there are sixteen different types of c-links, as the edge between $X$ and $A$ can go in either direction and have either sign yielding four possibilities, and similarly for the edge between $X$ and $B$, for a total of $4 \cdot 4=16$. For each of these types of c-links we are interested in the frequencies of positive versus negative labels for the edge from $A$ to $B$. Figure 2 shows all the possible types of c-links, labeled $t_{1}-t_{16}$.

Now, for a particular type of c-link, we look at the set of all c-links $(A, B ; X)$ of this type, and ask: what fraction of the links from $A$ to $B$ in this set are positive? Moreover, how does this fraction compare to what one would expect from the generative baselines of the nodes $A$ and the receptive baselines of the nodes $B$ that are involved in the creation of these $A-B$ links? If we can quantify the answer to this question in our data, we can look for effects like we saw in our motivating example - there, in the case of positive links from $X$ to $A$ and $B$, we believed the likelihood of a positive $A-B$ edge should exceed the generative baseline of $A$ but should lie below the receptive baseline of $B$.

Let's consider a particular type $t$ of c-link, and suppose that $\left(A_{1}, B_{1} ; X_{1}\right),\left(A_{2}, B_{2} ; X_{2}\right), \ldots,\left(A_{k}, B_{k} ; X_{k}\right)$ is a list of all instances of this type $t$ of c-link in our data. We define the generative baseline for this type $t$ to be the sum of the generative baselines of all nodes $A_{i}$. This quantity is simply the expected number of positive edges we would get if we let each $A_{i}-B_{i}$ link form according to the generative baseline of $A_{i}$. We then define the generative surprise $s_{g}(t)$ for this type $t$ to be the (signed) number of standard deviations by which the actual number of positive $A_{i}-B_{i}$ edges in the data differs above or below this expectation. In other words, if the context provided by the node $X$ and its links with $A$ and
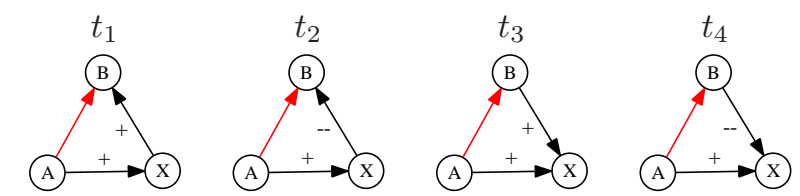

$t_{5}$
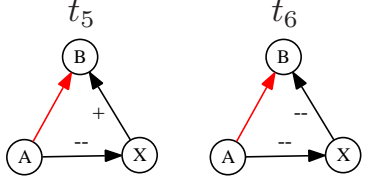

$t_{7}$

$t_{8}$
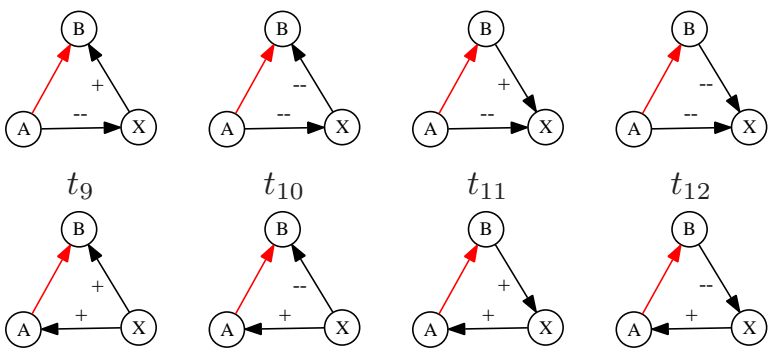

$t_{13}$
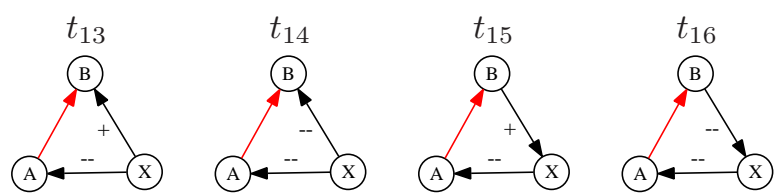

\begin{tabular}{c|rrrr|rrrr}
$t_{i}$ & count & $P(+)$ & $s_{g}$ & $s_{r}$ & $B_{g}$ & $B_{r}$ & $S_{g}$ & $S_{r}$ \\
\hline$t_{1}$ & 178,051 & 0.97 & 95.9 & 197.8 & $\checkmark$ & $\checkmark$ & $\checkmark$ & $\checkmark$ \\
$t_{2}$ & 45,797 & 0.54 & -151.3 & -229.9 & $\checkmark$ & $\checkmark$ & $\checkmark$ & $\circ$ \\
$t_{3}$ & 246,371 & 0.94 & 89.9 & 195.9 & $\checkmark$ & $\checkmark$ & $\circ$ & $\checkmark$ \\
$t_{4}$ & 25,384 & 0.89 & 1.8 & 44.9 & $\circ$ & $\circ$ & $\checkmark$ & $\checkmark$ \\
$t_{5}$ & 45,925 & 0.30 & 18.1 & -333.7 & $\circ$ & $\checkmark$ & $\checkmark$ & $\checkmark$ \\
$t_{6}$ & 11,215 & 0.23 & -15.5 & -193.6 & $\circ$ & $\circ$ & $\checkmark$ & $\checkmark$ \\
$t_{7}$ & 36,184 & 0.14 & -53.1 & -357.3 & $\checkmark$ & $\checkmark$ & $\checkmark$ & $\checkmark$ \\
$t_{8}$ & 61,519 & 0.63 & 124.1 & -225.6 & $\checkmark$ & $\circ$ & $\checkmark$ & $\checkmark$ \\
$t_{9}$ & 338,238 & 0.82 & 207.0 & -239.5 & $\checkmark$ & $\circ$ & $\checkmark$ & $\checkmark$ \\
$t_{10}$ & 27,089 & 0.20 & -110.7 & -449.6 & $\checkmark$ & $\checkmark$ & $\checkmark$ & $\checkmark$ \\
$t_{11}$ & 35,093 & 0.53 & -7.4 & -260.1 & $\circ$ & $\circ$ & $\checkmark$ & $\checkmark$ \\
$t_{12}$ & 20,933 & 0.71 & 17.2 & -113.4 & $\circ$ & $\checkmark$ & $\checkmark$ & $\checkmark$ \\
$t_{13}$ & 14,305 & 0.79 & 23.5 & 24.0 & $\circ$ & $\circ$ & $\checkmark$ & $\checkmark$ \\
$t_{14}$ & 30,235 & 0.69 & -12.8 & -53.6 & $\circ$ & $\circ$ & $\checkmark$ & $\circ$ \\
$t_{15}$ & 17,189 & 0.76 & 6.4 & 24.0 & $\circ$ & $\circ$ & $\circ$ & $\checkmark$ \\
$t_{16}$ & 4,133 & 0.77 & 11.9 & -2.6 & $\checkmark$ & $\circ$ & $\checkmark$ & $\circ$ \\
\hline \hline
\end{tabular}

Figure 2. Top: All contexts $(A, B ; X)$. Red edge is the edge that closes the triad. Bottom: Surprise values and predictions based on the competing theories of structural balance and status. $t_{i}$ refers to triad contexts above; Count: number of contexts $t_{i} ; P(+)$ : prob. that closing red edge is positive; $s_{g}$ : surprise of edge initiator giving a positive edge; $s_{r}$ : surprise of edge destination receiving a positive edge; $B_{g}:$ consistency of balance with generative surprise; $B_{r}$ : consistency of balance with receptive surprise; $S_{g}$ : consistency of status with generative surprise; $S_{r}$ : consistency of status with receptive surprise.

$B$ had no effect on the sign of the $A-B$ link being formed, so that each node $A_{i}$ simply drew the sign of her link to $B_{i}$ according to her generative baseline, then we should expect to see a generative surprise of 0 for this type $t$.

We set up the corresponding definitions for the nodes $B_{i}$ as the recipients of the links. We define the receptive baseline for this type $t$ of c-link to be the sum of the receptive baselines of all nodes $B_{i}$, and we define the receptive surprise $s_{r}(t)$ to be the (signed) number of standard deviations by which the actual number of positive $A_{i}-B_{i}$ edges in the data differs above or below this expectation.

Incorporating the Role of Status. Finally, we bring the role of status into this theory. For this, it is useful to return once more to our motivating example. When a player $X$ on our hypothetical soccer team gave positive evaluations to both $A$ 
and $B$, we concluded - in the absence of any further information - that $A$ and $B$ were likely to have above-average status. We would have concluded the same thing had $A$ and $B$ given negative evaluations to $X$. On the other hand, if $X$ had evaluated $A$ and $B$ negatively, or had they evaluated $X$ positively, then we should have concluded that $A$ and $B$ were more likely than not to have below-average status.

This reasoning provides a way to assign status values to $A$ and $B$ in any type of c-link, as follows. We first assign the node $X$ a status of 0 . Then, if $X$ links positively to $A$, or $A$ links negatively to $X$, we assign $A$ a status of 1 ; otherwise, we assign $A$ a status of -1 . We use the same rule for assigning a status of 1 or -1 to $B$. Thus we say that the generative surprise for type $t$ is consistent with status if $B$ 's status has the same sign as the generative surprise: in this case, high-status recipients $B$ receive more positive evaluations than would be expected from the generative baseline of the node $A$ producing the link. We say that the receptive surprise for type $t$ is consistent with status if $A$ 's status has the opposite sign from the receptive surprise: high-status generators of links $A$ produce fewer positive evaluations than would be expected from the receptive baseline of the node $B$ receiving the link.

\section{Results}

We now evaluate the predictions of these theories on the two networks, Epinions and Wikipedia, for which we have data on the exact order in which the links were created. We focus our discussion on Epinions, for which the data is an order of magnitude larger; the results are quite similar on the smaller Wikipedia dataset, with differences that we note below.

We consider four theories to explain the signs of the links that are produced. The first two are the consistency of status with generative and receptive surprise, as just defined. The other two theories are the analogous forms of consistency with Heider's original notion of balance. Specifically, we say that Heider balance is consistent with generative surprise for a particular c-link type if the sign of the generative surprise is equal to the sign of the edge as predicted by balance. Analogously, we say that Heider balance is consistent with receptive surprise for a particular c-link type if the sign of the receptive surprise is equal to the sign of the edge as predicted by balance.

We find that the predictions of status with respect to both generative and receptive surprise perform much better against the data that the predictions of structural balance. Indeed, status is consistent with generative and receptive surprise on the vast majority of c-link types; as shown in Figure 2, it is consistent on 14 and 13 types respectively. This includes the case of joint endorsement (type $t_{9}$ in Figure 2) - which is in fact the most abundant type of c-link in the data - and also includes the natural counterpart of joint endorsement, in which $A$ and $B$ each link negatively to $X$ (type $t_{8}$ ). It also includes the case of a positive cycle (type $t_{11}$ ), discussed earlier as well. ${ }^{1}$

\footnotetext{
${ }^{1}$ On the Wikipedia dataset, the results for receptive surprise are almost identical; status is consistent with receptive surprise on all clink types except for the same three exceptional cases as Epinions,
}

Structural balance is a much weaker fit to the data: balance is consistent with generative surprise for only 8 of the 16 types of c-links, and consistent with receptive surprise for only 7 of the 16. We also evaluated consistency of generative and receptive surprise with respect to Davis's weaker notion of balance, with similar results. The one subtlety in evaluating the data with respect to Davis balance is that Davis's theory does not predict the sign of the $A-B$ edge in c-link types where the two existing edges with $X$ are both negative $\left(t_{6}, t_{8}, t_{14}\right.$, and $\left.t_{16}\right)$ : for these triads, either a positive or a negative $A-B$ link would be consistent with Davis's theory, and so no prediction can be made. Thus, we evaluate consistency of Davis balance with respect to generative and receptive surprise only on the remaining 12 c-link types; here, we find consistency in 6 and 7 of the 12 cases respectively. This too is much weaker than the predictions of status.

We also consider the structure of the cases in which status theory fails to make a correct prediction, analyzing the possible strengthenings of the theory that this might hint at. First, we observe that one of the two c-link types where status is inconsistent with generative surprise is the configuration in which $A$ and $B$ each link positively to $X$ (type $t_{3}$ ). This is one of the most basic settings for structural balance in Heider's work: if two people each like a third party, then one should expect them to have positive relations. It thus suggests where users of these systems may be relying on balance-based reasoning more than status-based reasoning.

We can get further insights from the cases where status theory is inconsistent with the data. In particular, the $16 \mathrm{c}$-link types can be divided into four groups of four each, based on whether $A$ has high or low status relative to $X$, and whether $B$ has high or low status relative to $X$. In looking at where status theory makes mistakes, it is almost exclusively on the c-link types where $A$ and $B$ are both posited to have low status relative to $X$. This corresponds to the types $t_{2}, t_{3}, t_{14}$, and $t_{15}$; we observe that with respect to generative surprise, both of status theory's mistakes occur on types of this form, and with respect to receptive surprise, two of status theory's three mistakes occur on types of this form.

Even further, the mistakes of status with respect to generative and receptive surprise on these types constitute natural "duals" to each other. Note first that if we reverse both the direction and the sign of an edge, we preserve the status relation of the two endpoints (e.g. a positive link from $A$ to $X$ or a negative link from $X$ to $A$ both suggest that $A$ has lower status than $X$ ). With this in mind, we observe that if we take the types $t_{3}$ and $t_{15}$ on which status theory makes its two mistakes with respect to generative surprise, and we reverse the directions and signs of both edges involving $X$, we get the c-link types $t_{2}$ and $t_{14}$ - these are the other two c-link types where $A$ and $B$ have low status relative to $X$, and they are two of the three types on which status theory makes mistakes with respect to receptive surprise.

\footnotetext{
$t_{2}, t_{14}$, and $t_{16}$, and one more: $t_{4}$. We find this close alignment quite surprising given the very different kinds of activities that the Epinions and Wikipedia links represent. On Wikipedia, status is also consistent with generative surprise on 12 of the 16 triad types, though here the types where there is inconsistency differ more from Epinions: $t_{14}$ (as in Epinions), $t_{5}, t_{8}$, and $t_{16}$.
} 


\begin{tabular}{l|r|r} 
Epinions & Count & Probability \\
\hline \hline$P(+\mid+)$ & 38,415 & 0.969 \\
$P(-\mid+)$ & 1,204 & 0.031 \\
$P(+\mid-)$ & 1,192 & 0.692 \\
$P(-\mid-)$ & 560 & 0.308 \\
\hline Wikipedia & Count & Fraction \\
\hline \hline$P(+\mid+)$ & 2,509 & 0.945 \\
$P(-\mid+)$ & 145 & 0.055 \\
$P(+\mid-)$ & 193 & 0.706 \\
$P(-\mid-)$ & 80 & 0.294
\end{tabular}

Table 4. Edge reciprocation. Given that the first edge was of sign $X$ $P(Y \mid X)$ give the probability that reciprocated edge is $Y$.

It is thus natural to conjecture that the use of signed links deviates most strongly from status theory when $A$ is predicted to impute low status to both herself and $B$. Now that this behavioral asymmetry has been identified in the data, via our formulation of this theory, developing a more refined theory of status that takes this asymmetry into account is an interesting direction for further work.

\section{RECIPROCATION OF DIRECTED EDGES}

Thus far we have found that balance theory is a reasonable approximation to the structure of signed networks when they are viewed as undirected graphs, while status theory better captures many of the properties when the networks are viewed in more detail as directed graphs that grow over time.

To understand the boundary between these two theories and where they apply, it is interesting to consider a particular subset of these networks where the directed edges are used to create symmetric relationships. This subset is the collection of edges that are reciprocal: cases in which there are two nodes $A$ and $B$ such that $A$ links to $B$ and $B$ also links to $A$. (If the $B-A$ link forms after the $A-B$ link, we say that $B$ reciprocates the link to $A$.) In our data, only about $3-5 \%$ of the edges represent the reciprocation of an existing link, so this is far from being a dominant mode of link creation on these systems. But it is an interesting mode of link creation, in that it represents a directly mutual relationship between two individuals $A$ and $B$, which is the setting in which balance theory has been more relevant to our earlier analyses.

Our findings for this type of linking suggest the following intuitively natural picture: in the relatively small portion of these networks where mutual back-and-forth interaction takes place, the principles of balance are more pronounced than they are in the larger portions of the networks where signed linking (and hence evaluation of others) takes place asymmetrically. In other words, users treat each other differently in the context of back-and-forth interaction than when they are using links to refer to others who do not link back.

We summarize the results in Table 4. First, we find that the reciprocation of positive $A-B$ edges is closely consistent with balance rather than status, while the reciprocation of negative edges seems to follow a hybrid of the two principles. Specifically, if $A$ links positively to $B$, then balance predicts that $B$ should link positively to $A$, while status predicts that $B$ has the higher status and should therefore link negatively to $A$. For the two systems in which we have data on the order of edge creation - Epinions and Wikipedia we find that the data clearly supports the balance interpreta-

\begin{tabular}{l||r|r|r|r} 
Epinions & Triads & $P(\mathrm{RSS})$ & $P(+\mid+)$ & $P(-\mid-)$ \\
\hline Balanced & 348,538 & 0.929 & 0.941 & 0.688 \\
Unbalanced & 74,860 & 0.788 & 0.834 & 0.676 \\
\hline \hline Wikipedia & Triads & $P(\mathrm{RSS})$ & $P(+\mid+)$ & $P(-\mid-)$ \\
\hline Balanced & 53,973 & 0.912 & 0.934 & 0.336 \\
Unbalanced & 13,542 & 0.661 & 0.878 & 0.195
\end{tabular}

Table 5. Edge reciprocation in balanced and unbalanced triads. Triads: number of balanced/unbalanced triads in the network where one of the edges was reciprocated. $P(\mathrm{RSS})$ : probability that the reciprocated edge is of the same sign. $P(+\mid+)$ : probability that the + edge is later reciprocated with a plus. $P(-\mid-)$ : probability that the - edge is reciprocated with a minus.

tion, as shown in Table 4 . When a $B$ - $A$ link reciprocates a positive $A-B$ link, this $B-A$ link is positive well over $90 \%$ of the time - much higher than the roughly $80 \%$ fraction of positive links in the system as a whole.

Reciprocation of a negative $A-B$ link, on the other hand, displays ingredients of both theories. When $A$ links negatively to $B$ and $B$ subsequently links to $A$, balance theory predicts a negative link while status theory predicts a positive one (since $A$ should have higher status). In the data, such $B-A$ links are positive roughly $70 \%$ of the time. This shows that users respond to a negative link with a positive link a majority of the time, but still at a rate below the $80 \%$ fraction of positive links in the system as a whole, suggesting a deviation in the direction of the balanced-based interpretation.

From Table 4, it is also interesting to observe how similar the probabilities for all kinds of reciprocation are between the two systems Epinions and Wikipedia. This is particularly striking given how different the level of public display of link signs is on these systems; it suggests that these rates of alignment in the signs are being driven by forces that may be relatively robust to the way in which link signs are presented.

\section{The Role of Triadic Structure in Reciprocation}

We now consider how reciprocation between $A$ and $B$ is affected by the context of $A$ and $B$ 's relationships to third nodes $X$. Specifically, suppose that an $A-B$ link is part of a directed triad in which each of $A$ and $B$ has a link to or from a node $X$. Now, $B$ reciprocates the link to $A$. As indicated in Table 5, we find that the $B$ - $A$ link is significantly more likely to have the same sign as the $A-B$ link when the original triad on $A-B-X$ (viewed as an undirected triad) is structurally balanced. In other words, when the initial $A-B$ $X$ triad is unbalanced, there is more of a latent tendency for $B$ to "reverse the sign" when she links back to $A$. The effect holds in all cases; it is more pronounced in Wikipedia than in Epinions, which is interesting given the difference in how public the edge signs are.

This result further indicates how balance-based effects seem to be at work in the portions of the networks where directed edges point in both directions, reinforcing mutual relationships. We conjecture that this tension between mutuality and asymmetry in different parts of the network will be relevant in understanding more deeply the interplay between status and balance effects in shaping the formation of links.

\section{FURTHER STRUCTURAL ANALYSIS OF SIGNED LINKS}

Finally, we explore some additional connections between network structure and the signs of links, focusing on the em- 
beddedness of edges and on the subgraphs consisting only of positive links and only of negative links. For these structural results, we analyze the networks as undirected graphs.

\section{Embeddedness of positive and negative ties}

We begin by trying to characterize the parts of the network in which positive ties are more likely to occur. Roughly, we find that positive ties are more likely to be clumped together, while negative ties tend to act more like bridges between islands of positive ties.

We explore this issue in Figure 3 by plotting the probability that an edge is positive as a function of its embeddedness , i.e., the number of common neighbors that its endpoints have [12], or equivalently, the number of distinct triads the edge participates in. For each dataset we plot two curves. In green, we show the results of a random-shuffling baseline - the sign probability we would get as a function of embeddedness if edge signs were determined randomly and independently with probability $p$ for each edge. As is clear, there is no dependence here between an edge's sign and its embeddedness, so the green curve is approximately flat.

However, in the real data (red) we see a completely different picture. Edges that are not well embedded (with endpoints having fewer than around 10 shared neighbors) tend to be more negative than expected based on the background probability $p$ of positive ties. However, as an edge is more embedded (participating in more triads) it tends to be increasingly positive. That is, a link is significantly more likely to be positive when its two endpoints have multiple neighbors (of either sign) in common. These findings are consistent across all three datasets. This suggests that positive edges tend to occur in better embedded (densely linked) groups of nodes, while negative edges tend to participate in fewer triangles, which indicates that they act as connections between the well-embedded sets of positive ties.

As mentioned in the Introduction, this observation is not part of the formulation of balance theory (and does not follow from it), but it is consistent with the notion from socialcapital theory of embedded edges being more "on display" $[3,5]$. Moreover, among our three datasets, this phenomenon is most pronounced for the Wikipedia voting data. This is also the only one of the three sites where the social relations are explicitly displayed to a broad set of users - thus putting the relations even more highly on display. Thus these results are particularly well explained in terms of implicit pressure to remain positive.

\section{All-Positive and All-Negative Networks}

To explore further the different roles played by positive and negative links in these networks, we study the sub-networks composed exclusively of the positive links and exclusively of the negative links. That is, we define the all-positive network to be the subgraph consisting only of the positive links, and the all-negative network to be the subgraph consisting only of the negative links. We also compare these to randomized baselines, in which we first randomly shuffle the edge signs in the full network, and then extract the all-positive and allnegative networks from these shuffled versions.

\begin{tabular}{l||r|r||r|r||r|r}
\multicolumn{1}{c||}{} & \multicolumn{2}{c}{ Size } & \multicolumn{2}{c|}{ Clustering } & \multicolumn{2}{c}{ Component } \\
& Nodes & Edges & Real & Rnd & Real & Rnd \\
\hline \hline Epinions: - & 119,090 & 123,602 & 0.012 & 0.022 & 0.308 & 0.334 \\
Epinions: + & 119,090 & 717,027 & 0.093 & 0.077 & 0.815 & 0.870 \\
\hline Slashdot: - & 82,144 & 124,130 & 0.005 & 0.010 & 0.423 & 0.524 \\
Slashdot: + & 82,144 & 425,072 & 0.025 & 0.022 & 0.906 & 0.909 \\
\hline Wikipedia: - & 7,115 & 21,984 & 0.028 & 0.031 & 0.583 & 0.612 \\
Wikipedia: + & 7,115 & 81,705 & 0.130 & 0.103 & 0.870 & 0.918
\end{tabular}

Table 6. Networks composed of only positive (negative) edges. Real: network induced on the positive (negative) edges. Rnd: network where edge signs are randomly permuted. Clustering: fraction of closed triads (closed triads divided by number of length 2 paths) Component: fraction of nodes in the largest connected component.

Table 6 summarizes several structural properties of these networks and their randomized variants. First, we consider the amount of clustering, defined as the fraction of $A-B-C$ paths in which the $A-C$ edge is also present (thus forming a "closed"triad $A-B-C$ ). In all three datasets, we find that the all-positive networks have significantly higher clustering than their randomized counterparts, and the all-negative networks have significantly lower clustering. This further reinforces the observation that positive edges tend to occur in clumps, while negative edges tend to span clusters.

Interestingly, both the all-positive and all-negative networks are less well-connected than expected, in the sense that their largest connected components are smaller than those of their randomized counterparts. While this may seem initially counterintuitive, one possible interpretation is as follows. The giant components of real social networks are believed to consist of densely connected clusters linked by less embedded ties $[11,19]$. The all-positive and all-negative networks in the real (rather than randomized) datasets are each biased toward one side of this balance: the all-positive networks have dense clusters without the bridging provided by less embedded ties, while the all-negative networks lack a sufficient abundance of dense clusters to sustain a large component.

We also consider the fraction of nodes that are outliers with respect to in- and out-degree in the all-positive and all-negative networks - with degrees exceeding twice the mean for the network. (For reasons of space, these numerical results are not shown in the table.) These outlier fractions remain largely unchanged when the edge signs are randomized, with two exceptions that each hint at interesting conclusions for the effects of displaying signed edges to users. First, the fraction of outliers for positive in-degree is higher than expected on Wikipedia, where edge signs are more public. This suggests a possible tendency for an excess of users to conform to already positive voting outcomes. Second, the fraction of outliers for negative out-degree is lower than expected on Epinions and Slashdot, where edge signs are less public. This is a bit more surprising; it suggests that despite the less public nature of the signs, there are fewer people who are prolific in their negative evaluations - either because the dynamics of these sites suppresses this type of people, or because they are not attracting people who engage in it.

\section{CONCLUSION}

Social networks underlying current social media sites often reflect a mixture of positive and negative links. Here we have investigated two theories of signed social networks balance and status. Balance is a classical theory from so- 


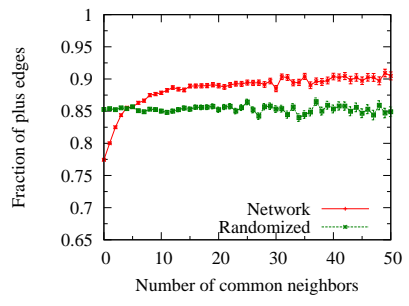

(a) Epinions

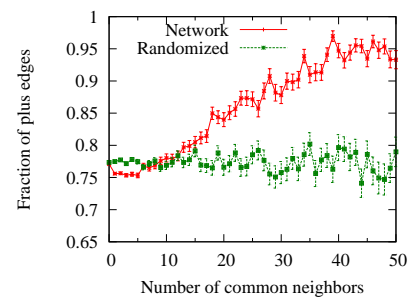

(b) Slashdot

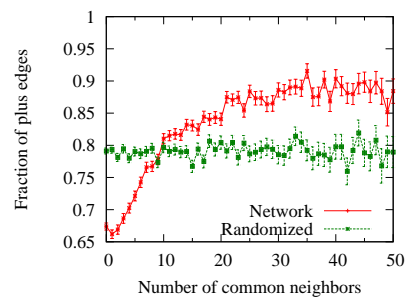

(c) Wikipedia cial psychology, which in its strongest form postulates that when considering the relationships between three people, either only one or all three of the relations should be positive. Status is a theory of directed signed networks which postulates that when person $A$ makes a positive link to person $B$, then $A$ is asserting that $B$ has higher status - with a negative link from $A$ analogously implying that $A$ believes $B$ has lower status. These two theories make different predictions for the frequency of different patterns of signed links in a social network. On networks derived from Epinions, Slashdot, and Wikipedia, we find that each model predicts certain kinds of social relationships, and that there is strong consistency in how the models fit the data across these three relatively different settings. Moreover, differences in results between the datasets highlight some interesting aspects of how the sites present information.

We have discussed the central interpretations of our findings, and here we briefly review some of the most salient. When the networks are viewed as undirected graphs, we find strong evidence for a weak form of structural balance, observing that in all three datasets triangles with exactly two positive signs are massively underrepresented in the data relative to chance, while triangles with three positive edges are overrepresented. We further find that a link is significantly more likely to be positive when its two endpoints have multiple neighbors (of either sign) in common - a finding that connects balance with notions from the theory of social capital. This is particular pronounced for Wikipedia, where the signs of edges are also the most publicly prominent.

When the networks are viewed as directed graphs, on the other hand, incorporating the fact that each link is created by one individual to point to another, we find that many of the basic predictions of balance theory no longer apply. Instead, the signs of directed links closely follow the predictions of the theory of status we develop, in which inferences about the sign of a link from $A$ to $B$ can be drawn from the mutual relationships that $A$ and $B$ have to third parties $X$. The signs and directions of these relationships to $X$ provide information about the status levels of $A$ and $B$, which in turn accurately predict the deviations in the sign of their interaction from broader background distributions. Investigating different contexts for links, and the differences between one-way and reciprocated links, sheds further light on the subtle ways in which users of these systems draw on behaviors rooted in both balance and status when they link to one another.

\section{REFERENCES}

1. M. J. Brzozowski, T. Hogg, G. Szabó. Friends and foes: ideological social networking. Proc. ACM CHI, 2008.
2. M. Burke and R. Kraut. Mopping up: Modeling wikipedia promotion decisions. Proc. CSCW, 2008.

3. R. S. Burt. The network structure of social capital. Research in Organizational Studies, 22:345-423, 2000.

4. D. Cartwright, F. Harary. Structure balance: A generalization of Heider's theory. Psych. Rev. 63(1956).

5. J. S. Coleman. Social capital in the creation of human capital. American Journal of Sociology, 94(1988).

6. D. Cosley, D. Frankowski, S. Kiesler, L. Terveen, J. Riedl. How oversight improves member-maintained communities. Proc. CHI, 2005.

7. J. A. Davis. Clustering and structural balance in graphs. Human Relations, 20(2):181-187, 1967.

8. P. Doreian and A. Mrvar. A partitioning approach to structural balance. Social Networks, 18:149-168, 1996.

9. M. H. Fisek, J. Berger, R. Norman. Participation in heterogeneous and homogeneous groups: A theoretical intergration. American Journal of Sociology, 97(1991).

10. E. Gilbert, K. Karahalios. Predicting tie strength with social media. Proc. ACM CHI, 2009.

11. M. Granovetter. The strength of weak ties. American Journal of Sociology, 78:1360-1380, 1973.

12. M. Granovetter. Economic action and social structure: The problem of embeddedness. American Journal of Sociology, 91(3):481-510, Nov. 1985.

13. R. V. Guha, R. Kumar, P. Raghavan, A. Tomkins. Propagation of trust and distrust. Proc. WWW, 2004.

14. F. Heider. Attitudes and cognitive organization. Journal of Psychology, 21:107-112, 1946.

15. J. Kunegis, A. Lommatzsch, C. Bauckhage. The Slashdot Zoo: Mining a social network with negative edges. Proc. $W W W, 2009$.

16. C. Lampe, E. Johnston, P. Resnick. Follow the reader: Filtering comments on Slashdot. Proc. CHI, 2007.

17. D. Liben-Nowell, J. Kleinberg. The link-prediction problem for social networks. J. American Society for Information Science and Technology, 58(2007).

18. M. E. J. Newman. The structure and function of complex networks. SIAM Review, 45:167-256, 2003.

19. J.-P. Onnela, J. Saramaki, J. Hyvonen, G. Szabo, D. Lazer, K. Kaski, J. Kertesz, and A.-L. Barabasi. Structure and tie strengths in mobile communication networks. Proc. Natl. Acad. Sci. USA, 104(2007).

20. B. Pang and L. Lee. Opinion Mining and Sentinment Analysis. Now Publishers, 2008.

21. S. Wasserman, K. Faust. Social Network Analysis: Methods and Applications. Camb. U. Press, 1994.

22. D. Willer. Network Exchange Theory. Praeger, 1999. 